\title{
PRETEXT Stage 2 Hepatoblastoma
}

National Cancer Institute

\section{Source}

National Cancer Institute. PRETEXT Stage 2 Hepatoblastoma. NCI Thesaurus. Code C7140.

An internationally developed presurgical anatomic staging system using imaging techniques: T umor involves 2 adjoining quadrants; 2 adjoining quadrants are free of tumor. (from PDQ 2004) 